\title{
Thrombocytopenia and Absent Radii Syndrome with Tetralogy of Fallot and Hypothyroidism
}

\author{
Manoj Kumar Sahu ${ }^{1}$ Dharmraj Singh ${ }^{1}$ Gaind Saurav ${ }^{1}$ Sarvesh Pal Singh ${ }^{1} \quad$ Palleti Rajashekar ${ }^{1}$
}

\author{
${ }^{1}$ Department of Cardiothoracic and Vascular Surgery, CN Centre, All \\ India Institute of Medical Sciences, New Delhi, India
}

\begin{abstract}
Address for correspondence Manoj K. Sahu, MD, DNB, Department of Cardiothoracic and Vascular Surgery, CN Centre, All India Institute of Medical Sciences, CTVS Office, 7th Floor, New Delhi 110029, India (e-mail: drmanojsahu@gmail.com).
\end{abstract}
Abstract
Keywords
- TAR syndrome
- tetralogy of Fallot
- intracardiac repair
- hypothyroidism

Thrombocytopenia and absent radii (TAR) syndrome is a rare genetic disorder characterized by congenital absence of both radii, presence of both the thumbs, and severe thrombocytopenia which resolves spontaneously in childhood. The incidence is 0.5 to 1 per 100,000 live births and this syndrome may be associated with anomalies of other organs like gastrointestinal (milk protein allergy), genitourinary, cerebral, cardiac, renal, and so forth. Involvement of endocrine system is not reported in the literature. We describe a case of TAR syndrome with tetralogy of Fallot and newly diagnosed hypothyroidism.

\section{Introduction}

Thrombocytopenia and absent radii syndrome (TAR) is rare with a probable autosomal recessive inheritance. This syndrome with its cardinal manifestations was first described by J. G. Hall et al in 1969. ${ }^{1}$ A report from Klopocki and colleagues ${ }^{2}$ suggested that a specific microdeletion of chromosome 1q21.1 is necessary, but in itself insufficient, to cause the TAR phenotype. This disorder is characterized by severe thrombocytopenia with potential for fatal bleeding episodes during infancy. Often TAR syndrome is associated with congenital deformities of other organ systems like gastrointestinal, genitourinary, cerebral, cardiac, renal, and so forth. ${ }^{1-6}$ Other characteristic findings include bilateral radial aplasia and presence of thumbs. TAR syndrome has no gender, ethnic, or racial predilection ${ }^{7}$ but some studies have reported females' predilection.?

We present a child with TAR syndrome with tetralogy of Fallot (TOF) who underwent intracardiac repair (ICR). The child manifested features of hypothyroidism postoperatively which was a new find with this syndrome, not reported previously.

\section{Case Report}

A 4-year-old male child weighing $12 \mathrm{~kg}$ was admitted to our hospital for surgical repair of TOF. This was the second child born to a nonconsanguineous marriage at full term, a normal vaginal delivery in the hospital. There was no history of birth trauma or similar illness in the other sibling or family.
There was no unprescribed medication during pregnancy. The antenatal period was uneventful on regular checkup but ultrasonography was not done due to lack of facilities. At birth the child was noted to have bilateral upper limb deformity with shortened and radial deviation of forearms (-Fig. 1). There were no facial or lower limb deformity, no organomegaly and other systems were grossly normal. Blood chemistry was mostly unremarkable except polycythemia (hemoglobin $17 \mathrm{~g} / \mathrm{dL}$ ) and thrombocytopenia $44,000 / \mathrm{mm}^{3}$. Radiograph of bilateral upper limbs showed absence of both the radius and presence of both the thumbs ( - Fig. 2); ultrasonogram of abdomen and pelvis showed no abnormality. Echocardiography confirmed the diagnosis of TOF. There was no history of bleeding diathesis and clinical examination did not reveal hematoma, petechiae, or echymotic patches.

With these findings, a diagnosis of TAR syndrome with TOF was made. Genetic testing could not be done due to financial constraints.

ICR under general anesthesia remained uneventful, 6 units of random donor platelet concentrates were transfused post cardiopulmonary bypass after heparin reversal by protamine. The child was received in intensive care unit with stable hemodynamics with moderate inotropic support at dobutamine of $10 \mu \mathrm{g} / \mathrm{kg} / \mathrm{min}$, adrenaline of $0.1 \mu \mathrm{g} / \mathrm{kg} / \mathrm{min}$, and nitroglycerine at $0.3 \mu \mathrm{g} / \mathrm{kg} / \mathrm{min}$. Echocardiography revealed mild right ventricular dysfunction and no other intracardiac issues, the child was ventilated overnight and was extubated on the first postoperative day. But he was
DOI https://doi.org/ $10.1055 / \mathrm{s}-0040-1708075$ ISSN 2457-0206.
(0)2020 Official Publication of The Simulation Society (TSS), accredited by International Society of Cardiovascular Ultrasound (ISCU).
License terms

() (1) $\Theta \circledast$ 


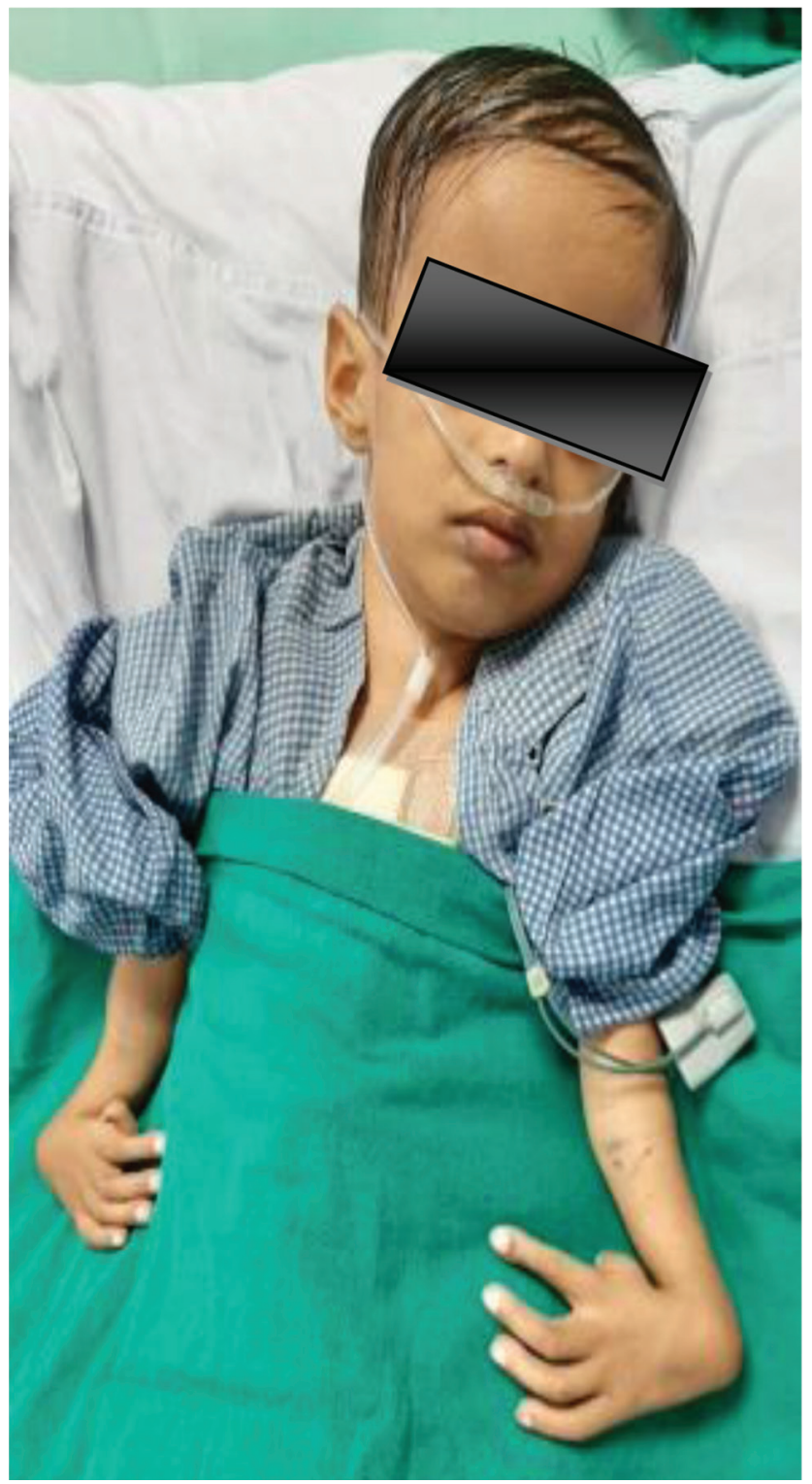

Fig. 1 Bilateral absent radius, radial club hand.

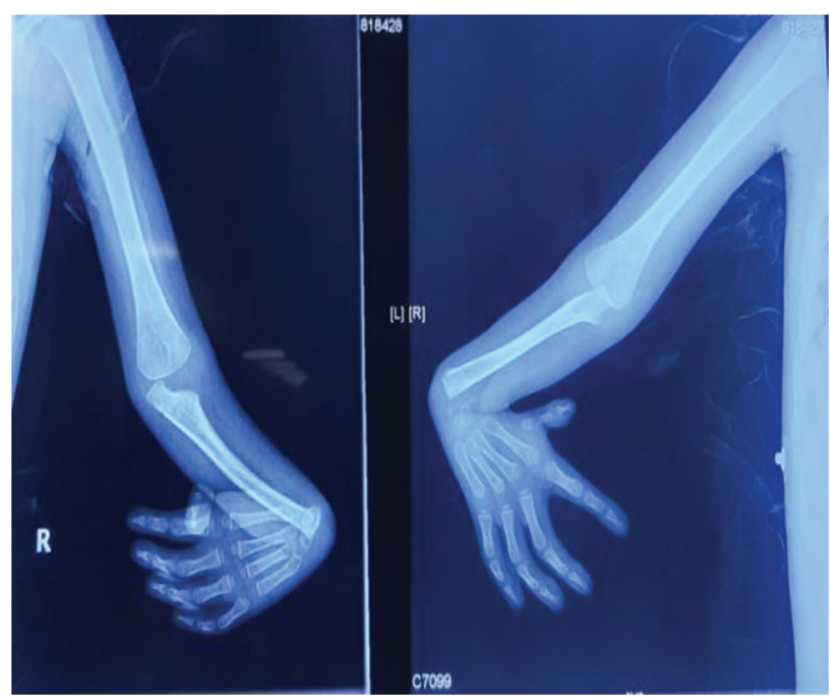

Fig. 2 Radiograph of both the upper limbs showing congenital absence of both the radius and presence of both the thumbs. reintubated $\sim 6$ hours after extubation because of inadequate breathing efforts, excessive lethargy, drowsiness, hypoventilation with raised partial pressure of carbon dioxide $\left(\mathrm{PaCO}_{2}\right)$, and decreased partial pressure of oxygen $\left(\mathrm{PaO}_{2}\right)$ in arterial blood.

Subsequently it became difficult to wean the child from the ventilatory support. All the other causes for drowsiness, hypoventilation, inadequate motor power like postanesthesia residual sedation, muscle relaxant effects, electrolyte and glucose imbalances, hypovolemia, and so forth were ruled out. Serum levels of triiodothyronin 3 (T3), triiodothyronin 4 (T4), and thyroid stimulating hormone (TSH) confirmed hypothyroidism (-Table 1). Oral thyroxin was begun at $30 \mu \mathrm{g}$ per day. The child required ionotropic support to maintain his hemodynamics till thyroid function became normal. The child's alertness and motor power improved after 72 hours; echocardiography showed good biventricular function, no other surgical issues. Then the child was separated from ventilator, weaned off ionotropic support gradually, and discharged from hospital on fifteenth day. The serum levels of T3, T4, and TSH with reference ranges on different postoperative days are shown in - Table $\mathbf{1}$.

\section{Discussion}

TAR syndrome, a presumed autosomal recessive disorder ${ }^{2}$ is associated with severe thrombocytopenia $\left(10-100 \times 10^{3} / \mathrm{mm}^{3}\right)$ since infancy, which resolves spontaneously as the child grows. ${ }^{3}$ Peripheral blood smear shows thrombocytopenia with normal platelet morphology, bone marrow study finds small, basophilic, vacuolated megakaryocytes. ${ }^{8}$ Bilateral radial aplasia and presence of thumbs in both hand ${ }^{3}$ are characteristic, making a sure clinical diagnosis of TAR. Lower limb skeletal abnormalities like hip dislocation, femoral and tibial torsion may be present but the case should be differentiated from Holt-Oram syndrome. Other syndromes like Fanconi anemia, Robert's syndrome, and RAPADILINO syndrome, ${ }^{3,4}$ and so forth, may need to be differentiated from TAR syndrome. No lower-limb anomalies were seen in our case. Our patient had congenital absence of both radii with bowing of the ulna and flexion deformity of the elbow and wrist joint ( - Fig. 1) with additional cardiac defect, that is, TOF. A similar case of TAR syndrome with TOF has been described earlier by Chetan Kumar et $\mathrm{al}^{9}$ but without any endocrine pathology. Treatment usually is directed toward raising the blood platelet levels by transfusing platelet concentrate and bring it to a safe level $\left(>100,000 / \mathrm{mm}^{3}\right)$ mainly during any major surgery.

Endocrine malfunction has not been reported with TAR syndrome previously. We in our case clinically suspected hypothyroidism due to excessive sleepiness, lethargy, inadequate motor power leading to respiratory failure, and reintubation on first postoperative day and subsequent inability to wean the child from respiratory support despite a fairly adequate cardiac function with moderate inotropic support. The case of hypothyroidism was confirmed from serum levels of T3, T4, TSH on postoperative day 3 (-Table $\mathbf{1}$ ). The child was treated with oral thyroxin and he improved thereafter. Then the separation from mechanical ventilation, gradual weaning off the inotropes was 
Table 1 The serum levels of T3, T4, and TSH on postoperative days 3, 5, and 7

\begin{tabular}{|l|l|l|l|l|}
\hline Postoperative days & T3 & T4 & TSH & Serum cortisol \\
\hline POD3 & 35.80 & 2.7 & 9.51 & 8.77 \\
\hline POD5 & 57.09 & 5.3 & 7.78 & - \\
\hline POD7 & 78.06 & 6.7 & 2.27 & - \\
\hline Reference range & $70-170 \mathrm{ng} / \mathrm{dL}$ & $4.5-12.5 \mu \mathrm{g} / \mathrm{dL}$ & $0.4-4 \mu \mathrm{LU} / \mathrm{dL}$ & $5-25 \mu \mathrm{g} / \mathrm{dL}$ \\
\hline
\end{tabular}

Abbreviations: POD, postoperative day; T3, triiodothyronin 3; T4, triiodothyronin 4; TSH, thyroid stimulating hormone.

possible. Oral thyroxin supplementation improves the cardiac index and reduces the inotropic requirement, duration of mechanical ventilation, and intensive care unit and hospital stays in infants post congenital cardiac surgery..$^{10}$

We ruled out the other causes of hypothyroidism like congenital hypothyroidism $(\mathrm{CH})$, thyroxine-binding globulin (TBG) deficiency, and euthyroid sick syndrome (ESS) in this case as follows:

This was not a case of $\mathrm{CH}$ as this child did not have a history of maternal thyroid deficiency nor the mother was on any supplementation for that. Infants with $\mathrm{CH}$ usually present within the first month of life with clinical signs of hypothyroidism like macroglossia, enlarged fontanelle, hypotonia, and decreased mental and physical activity and no such history/clinical features were present with this child. Also as per the American Academy of Pediatrics guidelines (infant with a low T4 concentration and TSH concentration greater than $40 \mathrm{mU} / \mathrm{L}$ is considered to have primary hypothyroidism $)^{11}$ our patient's TSH level was not suggestive of congenital hypothyroidism.

Thyroxine binding globulin deficiency as a differential diagnosis was also ruled out in our case from the thyroid function tests (TFTs). In patients with TBG deficiency TSH and free T4 levels are normal but total T4 levels are low. In our patient TSH levels were high and that decreased to normalcy with thyroxin supplementation.

Euthyroid sick syndrome is described as abnormal findings on thyroid function tests that occur in the setting of a nonthyroidal illness, which was another suspected clinical entity. In this variety the patient's TFTs are abnormal but they do not have clinical hypothyroidism. The TFTs here are very variable and the hormone levels normalize after the illness is cured. Our patient typically manifested the features of severe hypothyroidism, required thyroxin replacement and normalized the hormone levels with supplementation, and so excluded not having ESS.

\section{Conclusion}

Many congenital anomalies of other organ systems have been described to be associated with TAR syndrome, but endocrine dysfunction has not been reported earlier. Hypothyroidism was diagnosed for the first time in our case with TAR syndrome and the child recovered with thyroxin supplementation. This is a new clinical finding the clinicians must be aware of, even though it is not possible to label hypothyroidism as a new association with TAR syndrome and this requires further genetic studies and more reports.

\section{Informed Consent}

Informed and written consent was obtained from the father of the child for possible publication of the case.

\section{Funding}

No funds were received from anywhere for writing this case report.

\section{Conflict of Interest}

There was no conflict of interest among the authors.

\section{References}

1 Hall JG, Levin J, Kuhn JP. Ottenheimer EJ, van Berkum KA, McKusick VA. Thrombocytopenia with absent radius (TAR) Medicine (Baltimore) 1969;48(6):411-439

2 Klopocki E, Schulze H, Strauss G, et al. Complex inheritance pattern resembling autosomal recessive inheritance involving a microdeletion in thrombocytopenia-absent radius syndrome. Am J Hum Genet 2007;80(2):232-240

3 de Ybarrondo L, Barratt MS. Thrombocytopenia absent radius syndrome. Pediatr Rev 2011;32(9):399-400, discussion 400

4 Tsilou ET, Giri N, Weinstein S, Mueller C, Savage SA, Alter BP. Ocular and orbital manifestations of the inherited bone marrow failure syndromes: Fanconi anemia and dyskeratosis congenita. Ophthalmology 2010;117(3):615-622

5 Menghsol SC, Harris RD, Ornvold K. Thrombocytopenia and absent radii, TAR syndrome: report of cerebellar dysgenesis and newly identified cardiac and renal anomalies. (Letter) Am J Med Genet A 2003;123A(2):193-196

6 Williams J, Kim BY, Warrier R. An infant with abnormal upper extremity findings: a case report. Clin Pediatr (Phila) 2018;57(8):997-998

7 Alagbe OA, Alagbe AE, Onifade EO, Bello TO. Thrombocytopenia with absent radii (TAR) syndrome in a female neonate: a case report. Pan Afr Med J 2019;33:181

8 Go RS, Johnston KL. Acute myelogenous leukemia in an adult with thrombocytopenia with absent radii syndrome. Eur J Haematol 2003;70(4):246-248

9 Kumar C, Sharma D, Pandita A, Bhalerao S. Thrombocytopenia absent radius syndrome with Tetralogy of Fallot: a rare association. Int Med Case Rep J 2015;8:81-85

10 Talwar S. Bhoje AKhadagawat R, Chaturvedi P, Sreenivas $\mathrm{V}$, Makhija N. Oral thyroxin supplementation in infants undergoing cardiac surgery: a double-blind placebo-controlled randomized clinical trial. J Thorac Cardiovasc Surg 2018;156:1209-1217

11 Rose SR, Brown RS, Foley T, et al; American Academy of PediatricsSection on Endocrinology and Committee on Genetics, American Thyroid AssociationPublic Health Committee, Lawson Wilkins Pediatric Endocrine Society. Update of newborn screening and therapy for congenital hypothyroidism. Pediatrics 2006;117(6):2290-2303 
